\section{Small-angle X-ray scattering characterization of porous glasses}

\section{G. Walter ${ }^{a^{*}}$, R. Kranold ${ }^{a}$, D. Enke ${ }^{b}$ and G. Goerigk ${ }^{c}$}

${ }^{a}$ Department of Physics, Rostock University, Universitätsplatz 3, D-18051 Rostock, Germany, ${ }^{b}$ Institute of Technical Chemistry and Macromolecular Chemistry, Martin Luther University HalleWittenberg, Schloßberg 2, D-06108 Halle (Saale), Germany, 'Institute of Solid State Research, Jülich Research Center, P.O. Box 1913, D-52425 Jülich, Germany

Small-angle X-ray scattering has been used to examine porous glasses. The experiment and analysis of the extracted data are described with specific concern for the quantitative characterization of the pore structure. The techniques used are illustrated by the results obtained from two selected porous glasses. Small-angle X-ray scattering data of two series of novel porous glass systems are presented and discussed.

Keywords: small-angle X-ray scattering; glasses

\section{Introduction}

Porous glasses are usually prepared from $\mathrm{Na}_{2} \mathrm{O}-\mathrm{B}_{2} \mathrm{O}_{3}-\mathrm{SiO}_{2}$ glasses separated into two interconnected phases (Janowski \& Heyer, 1982; Mazurin \& Porai-Koshits, 1984). After acid leaching of the alkali borate-rich phase, a spongy silica skeleton is obtained (Fig. 1). The dissolved phase also contains silica which remains in the form of

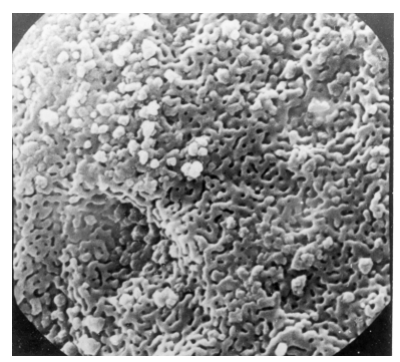

Fig. 1

Electron micrograph of a Vycor porous glass. The glass has an internal surface area of $40 \mathrm{~m}^{2} / \mathrm{g}$, the volume fraction of the pores is $w_{\mathrm{p}}=0.41$, and the average pore diameter amounts to $88 \mathrm{~nm}$.

finely dispersed colloidal silica in the cavities of the silica framework. The finely dispersed silica affects the pore structure and thus the properties of the resulting porous glass system. If undesired for the application of the final material, the colloidal silica is removed by treatment with alkaline solution. In this way, so-called Controlled-Pore Glass (CPG) has been prepared. Porous glasses have an internal surface area up to $300 \mathrm{~m}^{2} / \mathrm{g}$. The most important uses of CPG's are in biotechnology, membrane science, confined matter physics and as chemical sensors (see Refs. 1 - 5 in Enke et $a l .$, 2001). There has been considerable technological and scientific interest in knowing the details of the pore structure of the glasses. Small-angle X-ray scattering (SAXS) is an excellent technique for characterizing porous glasses (Schmidt, 1988).

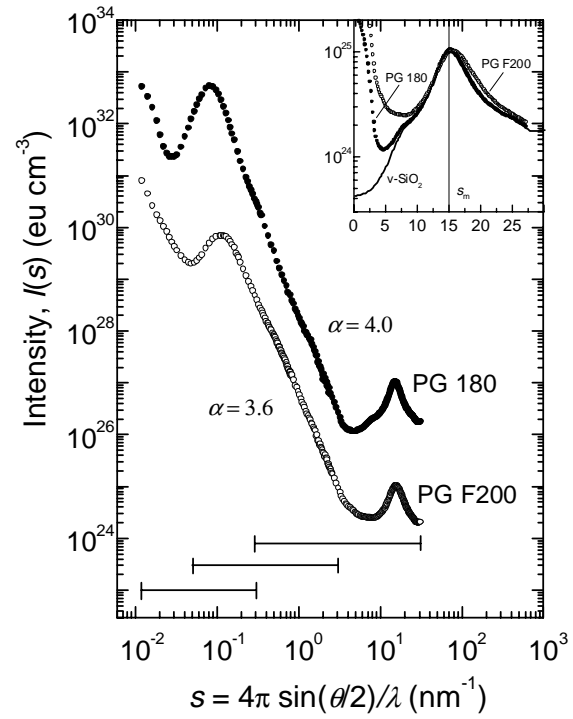

Fig. 2

The scattering curve for a macroporous glass (PG 180), which has been multiplied by $10^{2}$ for better visibility, and for a meso-macroporous glass (PG F200). $\alpha$ is the exponent of the power-law scattering (4). The horizontal bars in the lower part of the plot indicate the $s$ ranges covered with the three different SAXS systems used for recording the scattering from the glasses (see text). The inset is the enlargement of the diffraction peaks at large $s$ values compared with that of meltcooled vitreous $\mathrm{SiO}_{2}$.

\section{Method}

\subsection{SAXS measurements}

To cover a widely extended range of $s$ values, the scattering from porous glasses is recorded on different SAXS systems $(s=(4 \pi / \lambda)$ $\sin (\theta / 2)$ is the magnitude of the scattering vector where $\lambda$ is the $\mathrm{X}$ ray wavelength and $\theta$ is the scattering angle). The data of the curves shown in Fig. 2 were measured for $0.012 \leq s \leq 0.3 \mathrm{~nm}^{-1}$ with a Bonse-Hart camera at the University of Missouri-Columbia, for $0.05 \leq s \leq 3 \mathrm{~nm}^{-1}$ with the SAXS system at the National Center for Small-Angle Scattering Research at Oak Ridge, and for $0.3 \leq s \leq 31$ $\mathrm{nm}^{-1}$ with a Rigaku Denki low-angle X-ray goniometer at the University of Rostock. The three curves for a given sample were joined in the regions where they overlapped and converted into absolute units (electron units). A detailed description of the procedures used for data collection and corrections of the recorded intensities was given in Walter, Kranold, Göcke \& Enenkel (1991).

\subsection{Data analysis}

The most useful SAXS parameters for characterizing the average dimensions of the pore widths and the $\mathrm{SiO}_{2}$ skeleton are the mean intersection lengths, $d_{\mathrm{p}}$ and $d_{\mathrm{SiO}_{2}}$,of the pores and the $\mathrm{SiO}_{2}$ skeleton, respectively. The parameters can be determined using the equations (Porod, 1982) 


$$
\begin{aligned}
& d_{\mathrm{p}}=\frac{d_{\mathrm{c}}}{\left(1-w_{\mathrm{p}}\right)} \\
& d_{\mathrm{SiO}_{2}}=\frac{d_{\mathrm{c}}}{w_{\mathrm{p}}} .
\end{aligned}
$$

For infinitely long cylindrical pores, $d_{\mathrm{p}}$ represents the average pore diameter. In (1a) and (1b), $w_{\mathrm{p}}$ and $\left(1-w_{\mathrm{p}}\right)$ are the volume fractions of the two phases and $d_{\mathrm{c}}$ is the mean intersection length. The quantity $d_{\mathrm{c}}$ can be calculated from the first moment of the intersect distribution function (Porod, 1982), $A(l)$, according to the relation

$$
d_{\mathrm{c}}=\frac{d_{\mathrm{p}} d_{\mathrm{SiO}_{2}}}{d_{\mathrm{p}}+d_{\mathrm{SiO}_{2}}}=\int_{0}^{\infty} l A(l) \mathrm{d} l
$$

Fig. 3 presents the intersect distribution functions, $A(l)$, obtained for the two porous glasses the scattering curves of which are shown in Fig. 2. The $A(l)$ functions were derived from the correlation functions which were calculated from the ,infinite-slit' smeared SAXS curves using the method developed by Gerber \& Walter (1983). The $A(l)$ function calculated for PG F200 shows that this glass consists both of pores of $\sim 5 \mathrm{~nm}$ in size and a considerable number of larger pores. The volume fractions, $w_{\mathrm{p}}$ and $\left(1-w_{\mathrm{p}}\right)$, in (1a) and (1b) can be extracted from the mean-square electron density fluctuations that are related to the second moment of the intensity function, $I(s)$, expressed by

$$
\overline{(\Delta \rho)^{2}}=w_{\mathrm{p}}\left(1-w_{\mathrm{p}}\right) \cdot(\Delta \rho)^{2}=\frac{1}{2 \pi^{2}} \int_{0}^{\infty} s^{2} I(s) \mathrm{d} s .
$$

Further information on the constitution of the pore surface is obtained by means of the power-law scattering exponent, $\alpha$, that can be determined by approximating the decay of the intensity by the equation (Höhr, Neumann, Schmidt, Pfeifer \& Avnir, 1988)

$$
I(s)=C \cdot s^{-\alpha}
$$

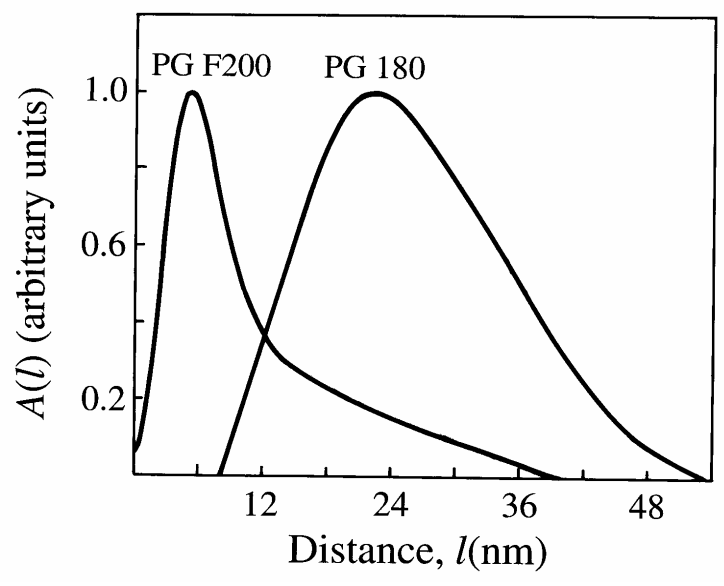

Fig. 3

The intersect distribution functions, $A(l)$, for the porous glasses whose intensity functions are shown in Fig. 2. The $A(l)$ functions were derived from the correlation functions which were calculated from the ,infinite-slit' smeared SAXS curves using the method developed by Gerber \& Walter (1983). where $C$ is a constant. Equation (4) was fitted to the curves shown in Fig. 2 in the interval $0.2<s<3 \mathrm{~nm}^{-1}$. The exponent $\alpha$ can be used to find the fractal dimension (Höhr, Neumann, Schmidt, Pfeifer \& Avnir, 1988), $D_{\mathrm{s}}$, of the pore bounderies given by

$$
\alpha=6-D_{\text {s }} \text {. }
$$

For fractal pore bounderies, $2 \leq D_{\mathrm{s}}<3$. When the pore bounderies are smooth, $D_{\mathrm{s}}=2$.

\section{Table 1}

Structural parameters for the porous glasses whose intensity functions are shown in Fig. 2.

$w_{\mathrm{p}}$ is the volume fraction of the pores; $d_{\mathrm{p}}$ and $d_{\mathrm{SiO}_{2}}$ are the mean intersection lengths of the pores and the $\mathrm{SiO}_{2}$ skeleton, respectively; $D_{\mathrm{s}}$ is the fractal dimension of the pore bounderies, and $s_{\mathrm{m}}$ is the position of the maximum of the diffraction peaks indicated in Fig. 2.

\begin{tabular}{lccccc}
\hline Sample & $w_{\mathrm{p}}$ & $d_{\mathrm{p}}$ & $d_{\mathrm{SiO}_{2}}$ & $D_{\mathrm{s}}$ & $\begin{array}{c}s_{\mathrm{m}} \\
(\mathrm{nm})\end{array}$ \\
\hline PG 180 & 0.42 & 40 & 56 & $2.0 \pm 0.1$ & 15.4 \\
PG F200 & 0.27 & 16 & 43 & $2.4 \pm 0.1$ & 15.8 \\
\hline
\end{tabular}

All parameters calculated for the two porous glasses under discussion (Fig. 2) are summarized in Table 1. From the values obtained for $D_{\mathrm{s}}$ it can be concluded that the surface of the pore channels in the macroporous glass (PG 180) is smooth, which results from the treatment with alkaline solution. On the contrary, in the meso-macroporous glass (PG F200), the surfaces are fractal, which may be caused by the finely dispersed $\mathrm{SiO}_{2}$ inside the larger pore channels or micropores in the walls of the channels owing to the chain-like distribution of $\mathrm{B}_{2} \mathrm{O}_{3}$ in the unleached silica-rich phase (Janowski \& Heyer, 1982; Mazurin \& Porai-Koshits, 1984).

An intense diffraction peak occurring in the intensity function at larger $s$ values is one signature of intermediate-range order in oxide glasses (Elliott, 1995; Gaskell \& Wallis, 1996). For melt-cooled vitreous $\mathrm{SiO}_{2}$, this prominent peak is observed in $I(s)$ at $s_{\mathrm{m}} \sim 15 \mathrm{~nm}^{-1}$ (Walter, Kranold, Göcke \& Enenkel, 1991). From the position (cf. Table 1) and the shape of the concerned peaks shown in the inset in Fig. 2 it can be concluded that the intermediate-range structure of the skeleton in PG 180 has great resemblance to that of melt-cooled vitreous $\mathrm{SiO}_{2}$, whereas the intermediate-range order of the silica skeleton and the finely dispersed $\mathrm{SiO}_{2}$ in the pore channels of the sample PG F200 can be expected to be different from the intermediate-range order of melt-cooled vitreous silica.

\section{Novel porous glass systems}

\subsection{Samples and techniques}

Two series of porous glasses, which are designated as $\mathrm{S} 1$ and $\mathrm{S} 2$, have been prepared from $\mathrm{Na}_{2} \mathrm{O}-\mathrm{B}_{2} \mathrm{O}_{3}-\mathrm{SiO}_{2}$ initial glasses, called GL 1 and GL 11 (Table 2). The initial glasses are identical in composition but strongly different in thermal history. In S1 the porous glasses, PGL $2-8$, were made by a process based on acid 
Table 2

Preparation characteristics for the two series, S1 and S2, of the glasses examined in this study.

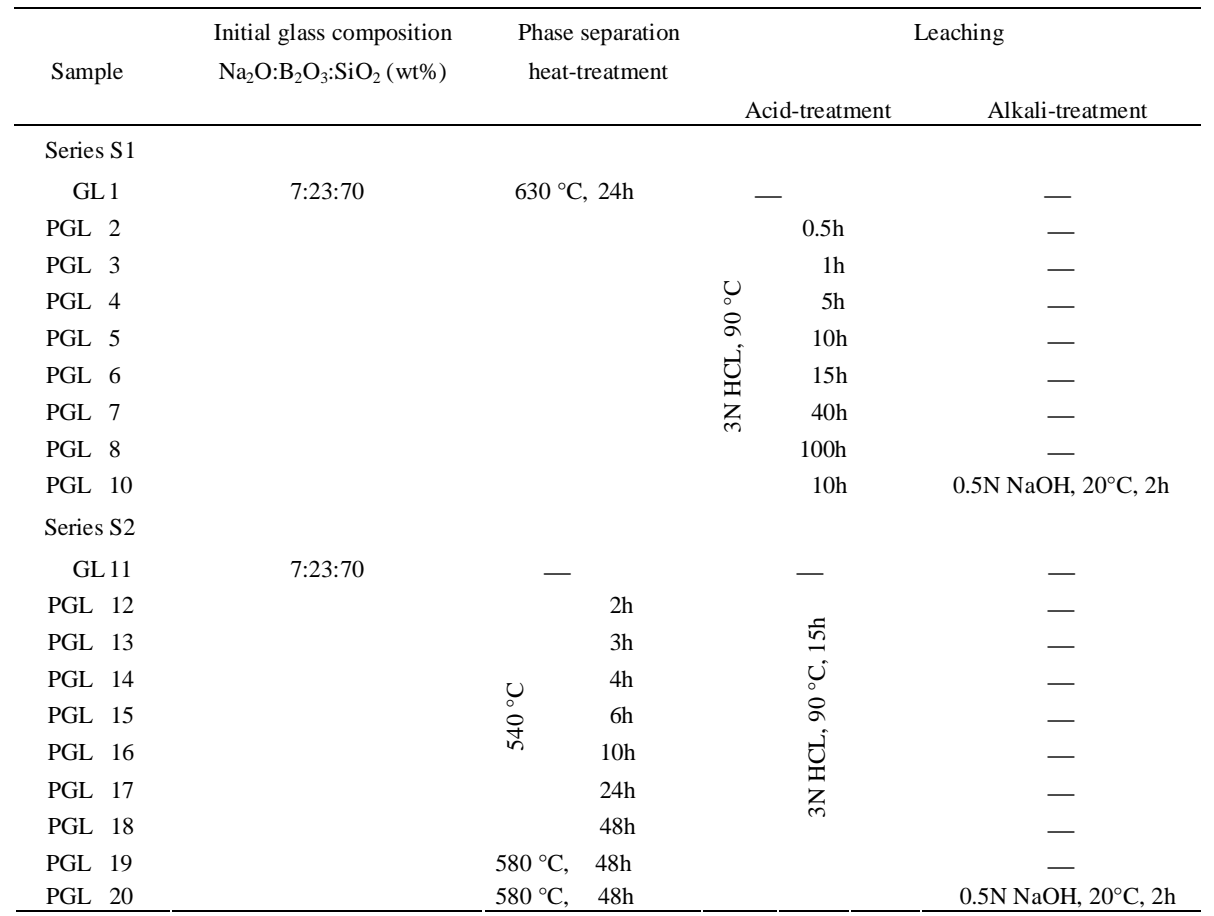

leaching of the phase separated glass, GL 1, for varying periods of time $(0.5-100 \mathrm{~h})$. The phase separated glass, GL 1, was produced in a heat treatment at $630{ }^{\circ} \mathrm{C}$ for $24 \mathrm{~h}$. Small portions of crystals formed on the surface during heating were removed by using $2 \mathrm{~N}$ $\mathrm{NaOH}$.

In contrast to $\mathrm{S} 1$, the initial glass, GL 11, in series S2 was not heat treated. Thus, the glass need not be expected to show phase separation. The porous glasses, PGL 12 - 19, were produced by subjecting GL 11 to heat treatment procedures with varying parameters and subsequent acid leaching.

In two of the samples, PGL 10 and 20, the finely dispersed silica, which remains in the pores after extracting the boron-rich phase, was dissolved by using alkali. Note that the pores in the samples PGL 12 - 18 do not contain finely dispersed silica, which is attributable to the low heat treatment temperature chosen.

The glass samples were in the form of grains of $100-200 \mu \mathrm{m}$ in diameter. The SAXS experiments were performed at beamline B1 at the Hamburg Synchrotron Radiation Laboratory at photon energy of $8294 \mathrm{eV}$. The distance between sample and detector was $3635 \mathrm{~mm}$. From this, scattering curves, $I(s)$, were measured with pinhole collimation in the interval $0.05 \leq s \leq 1.4 \mathrm{~nm}^{-1}$. The $I(s)$ functions experimentally obtained for the porous glasses including those for the initial glasses are shown in Figs. 4 and 5.

SAXS experiments to extend the $s$ range to $0.4 \leq s \leq 38 \mathrm{~nm}^{-1}$ (Rigaku Denki low-angle X-ray goniometer) are in progress. After completion of the measurements, the joined curves will be quantitatively evaluated as described and demonstrated in $\$ 2.2$.

\subsection{The scattered intensity}

The scattering effect observed for GL 1 and GL 11 (Figs. 4 and 5) is evidence that both initial glasses are phase separated, though GL 11 could be leached out only slightly $(13 \%)$. Note that the initial glass, GL 11, was not heat treated.

In series S1 (Fig. 4), the curves for the porous glasses PGL 2 - 8 characterize the effect of the duration of acid leaching of GL 1 (cf. Table 2). The glasses, PGL $2-8$, are micro-mesoporous, which is attributable to the finely dispersed silica that remains in the pores after the leaching of GL 1 is complete (Enke, Otto, Janowski, Heyer, Schwieger \& Gille, 2001). The changes of the pore structure are reflected by the evolution of the shoulder in the $I(s)$ functions at larger $s$ values. In the latest stage, the finely dispersed silica contained in PGL 5 was removed by a subsequent treatment using alkaline solutions. As a result a macroporous glass is obtained (PGL 10) indicated by the changes in the $s$ range where the shoulder in the intensity curves are observed for the micro-mesoporous glasses, PGL 2 - 8.

Series S2 comprises porous glasses having microporous and mesoporous constitution, which is achieved by a heat treatment of the initial glass GL 11 and subsequent acid treatment (cf. Table 2). The progressive phase separation produced by this procedure is characterized by the changes of the $I(s)$ functions shown in Fig. 5. In contrast to the series S1, the pores of the glasses PGL 12 - 18 do not contain finely dispersed silica after the leaching is complete. Thus, the changes in the curves obtained for PGL 12 - 18 are due to the 


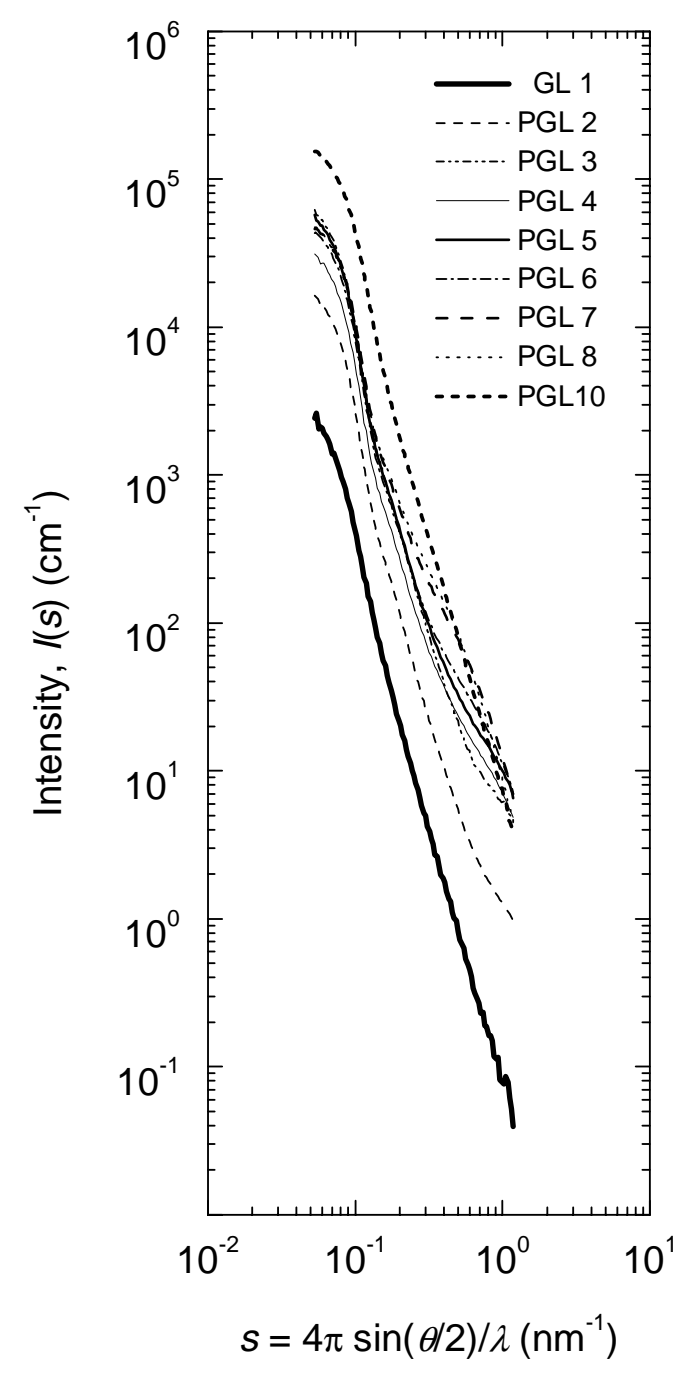

Fig. 4

The scattering curves for the glasses of series S1 (Table 2).

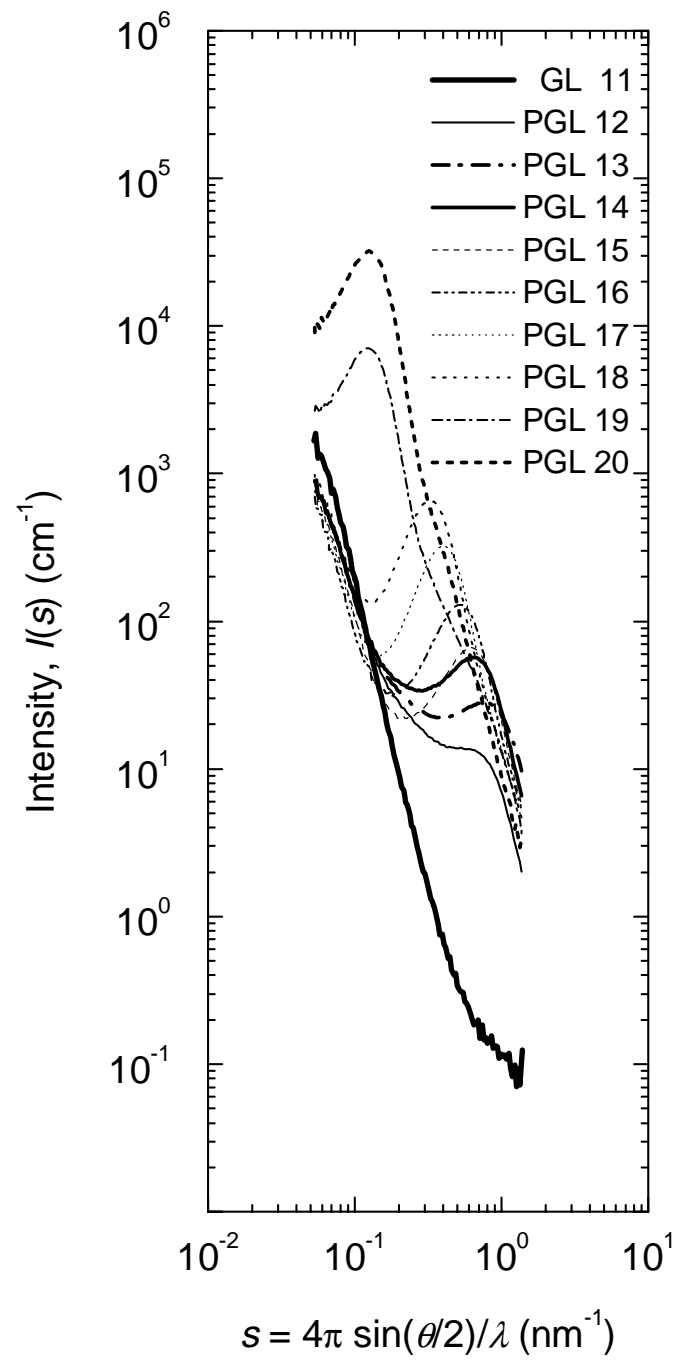

Fig. 5

The scattering curves for the glasses of series S2 (Table 2). growth of the pore channels indicated by the shift of the maxima to smaller $s$ values. The scattering observed in the range of small $s$ values is dominantly produced by the $\mathrm{SiO}_{2}$ skeleton.

The sample PGL 19 was heat treated at higher temperature than PGL 12 - 18 (cf. Table 2), and so it contains finely dispersed silica in the pores like PGL 2 - 8. The scattering curve for PGL 19 shows a maximum at $\mathrm{s}=0.126 \mathrm{~nm}^{-1}$ that is caused by some regularity in the pore system, whereas the finely dispersed silica contributes to the scattering at larger $s$ values indicated by the shoulder. This effect becomes obvious from the scattering curve obtained for PGL 20 that shows the maximum at the same position as PGL 19. In the glass PGL 20, the finely dispersed silica was removed by a subsequent treatment using alkaline solutions.

\section{Summary}

Characterizing the details of the pore structure of porous glasses is of most practical interest. For this purpose, small-angle X-ray scattering (SAXS) has proved to be very useful. To cover a widely extended range of $s$ values, the scattering from porous glasses has frequently to be recorded on different SAXS systems. The method provides structural parameters and functions with high accuracy which allow the quantitative characterization of the pore structure. Important parameters are the mean intersection lengths, which characterize the average dimensions of the pores and the skeleton, and the volume fractions of the two phases. Moreover, SAXS is among the few methods available for investigating the properties of the boundary 


\section{conference papers}

surfaces of the pores. As a result, it can be determined if the pore bounderies are smooth or fractal. Additional information is available from the diffraction peaks occurring in the intensity function at large $s$ values which can be taken as an indication of intermediate-range order.

\section{References}

Elliott, S. R. (1995). J. Non-Cryst. Solids 182, 40-48.

Enke, D., Otto, K., Janowski, F., Heyer, W., Schwieger, W. \& Gille, W. (2001). J. Mater. Sci. 36, 2349-2357.

Gaskell, P. H. \& Wallis, D. J. (1996). Phys. Rev. Lett. 76, 66-69.

Gerber, T. \& Walter, G. (1983). Stud. Biophys. 98, 47-52.

Höhr, A., Neumann, H.-B., Schmidt, P. W., Pfeifer, P. \& Avnir, D. (1988). Phys. Rev. B 38, 1462-1467.

Janowski, F. \& Heyer, W. (1982). Poröse Gläser-Herstellung, Eigenschaften und Anwendung. Leipzig: Deutscher Verlag für Grundstoffindustrie.

Mazurin, O. V. \& Porai-Koshits, E. A. (1984). Phase Separation in Glass. Amsterdam: North-Holland Physics Publishing.

Porod, G. (1982). In Small-Angle X-ray Scattering, edited by O. Glatter \& O. Kratky, pp. 17-51. New York, London: Academic Press.

Schmidt, P. W. (1988). In Characterization of Porous Solids. Proceedings of IUPAC Symposium, edited by K. K. Unger, J. Rouquerol, K. S. W. Sing \& H. Kral, pp. 35-48. Amsterdam: Elsevier.

Walter, G., Kranold, R., Göcke, W. \& Enenkel, N. (1991). J. Appl. Cryst. 24, 616-623. 